\title{
Research of hand tremor vibrations and interference with external mechanical oscillations source
}

\author{
M. Venslauskas*, E. Litvinas**, A. R. Juknevičius***, V. Ostaševičius**** \\ *Kaunas University of Technology, Studentu g. 56, 51424 Kaunas, Lithuania, E-mail: mantas.venslauskas@ktu.lt \\ **Kaunas University of Technology, Studentu g. 56, 51424 Kaunas, Lithuania, E-mail: litvinasedvinas@gmail.com \\ ***Kaunas University of Technology, Studentu g. 56, 51424 Kaunas, Lithuania, E-mail: andriusr.juknevicius@gmail.com \\ ***Kaunas University of Technology, Studentu g. 56, 51424 Kaunas, Lithuania, E-mail: vytautas.ostasevicius@ktu.lt
}

cross $^{\text {ref }}$ http://dx.doi.org/10.5755/j01.mech.22.5.16351

\section{Introduction}

Tens of millions people around the world suffers from hand tremor that are caused by essential tremor (ET) and Parkinson's disease (PD). More than 70\% of the population with upper limb tremor face serious difficulties in life. Functional disability and social inconvenience, contaminating daily life activities, such as writing, pouring, eating and so on makes hand tremor a trending problem in the society.

The roots of the essential tremor are not completely clear. It is thought that the defective electrical brain activity, that causes tremor, is processed through structure deep in the brain - thalamus. Thalamus coordinates and controls muscle activity. In case of Parkinson's disease neurons that are in an area of the brain, that controls movement, becomes impaired. Usually these neurons produce dopamine, but when they fail or become impaired, the production of this important brain chemical is reduced and the lack of this compound causes movement problems and tremor.

Various medications are available for the management of PD tremor. All of them are directed at easing symptoms and improving quality of life. At this time, no cure or method that reduce disease progression has been proven. Putative interactions between various applied drugs, recurrent appearance of non-motor features and treatment of motor symptoms ask for complex therapeutic interventions and careful drug titration. Reduction of dopaminergic drugs and hydration may sometimes be more beneficial than addition of further compounds [1]. The most commonly used drugs for ET include Propranolol, Primidone, but these drugs can cause serious side effects: fatigue, stuffy nose, or slow heartbeat, nausea and problems with walking, balance, and coordination. That makes drugs too complex and not so appropriate solution for ET and PD tremor problem.

Tremor issues are being solved by hardware as well. Liftware [2] is an electronical spoon/fork that is able to stabilize itself $70 \%$ for every tremor. Device is released on the market, but it solves only one problem. Imperial college of London students are developing glove with gyroscope which resist rotary movements to $90 \%$, but limits natural movements and is buzzing. Multiple patents of gyroscopic hand stabilization devices were published although device with this technology have never been released to market [3, 4]. In 2007 there were attempts to use electromechanical exoskeleton to reduce patient upper limb tremors [5]. Unfortunately machine is too big and heavy for everyday use.

Due to problem importance and relevance to society multiple research were done to analyse hand tremors.
Jen-Lin Yang and colleagues use laser line triangulation measurement method to analyse hand tremor resulting 4 - 6 $\mathrm{Hz}$ frequency of resting and $2-3 \mathrm{~Hz}$ postural tremors [6]. University of Delaware representatives use PHANToM platform for data collection resulting ellipsoidal tremor shape and two main frequencies for PD patients: $4.79 \mathrm{~Hz}$ and $8.78 \mathrm{~Hz}$ [7]. Meshack R.P. and K.E. Norman analyses tremors of 16 patients and concludes that the main frequency is $\sim 5.2 \mathrm{~Hz}$, amplitude varies from $0.5 \mathrm{~mm}$ to 4.28 $\mathrm{mm}[8]$.

Due to the lack of existing solution, our objective was to investigate tremor movement vectors, amplitudes and frequencies with photogrammetry on multiple patients, investigate the possibility to affect tremor by external mechanical oscillations and investigate how tremor affects different parts of patient arm.

8 patients with varied health conditions were tested. Second trial for one of the test subjects was required to analyse change of hand tremor vibration parameters over the time. Particular patient tremor was analysed 2 weeks after the first trial. In purpose of more general understanding of hand tremor, subjects with contrasting group of age and decease conditions were selected.

\section{Materials and methods}

In order to capture hand tremor vibrations, photogrammetry was used. Experiments were performed at the Laboratory of Biomechanics of the Mechatronic Institute at Kaunas University of Technology. The experiment was conducted using a ProReflex MCU 500 Type 170241 camera (Fig. 1) with Qualisys Track Manager Software. The ProReflex MCU uses a $680 \times 500$ pixel CCD image sensor. Usage of CCD technology results in low-noise data compared to a higher resolution CMOS sensor, which has a considerably

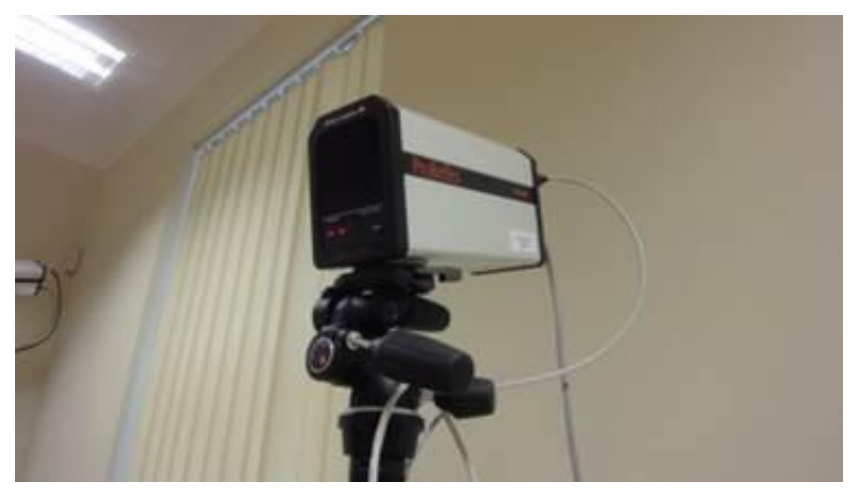

Fig. 1 ProReflex MCU 500 Type 170241 camera 
higher level of pixel noise. By using a patented sub-pixel interpolation algorithm, the effective resolution of the ProReflex MCU is $20000 \times 15000$ subpixels in a normal set-up, result the ProReflex MCU to detect motions as small as 50 microns [9].

Multiple parts of subject arm were tracked to observe possible tremor vibration differences. After investigating tremor visually, these upper limb investigation points were selected: shoulder, elbow, wrist, small finger knuckle, small fingernail, index finger knuckle, index finger nail. These points are close to joints, where the biggest vibrations can be seen. Therefore, light reflective markers were placed on these points (Fig. 2).

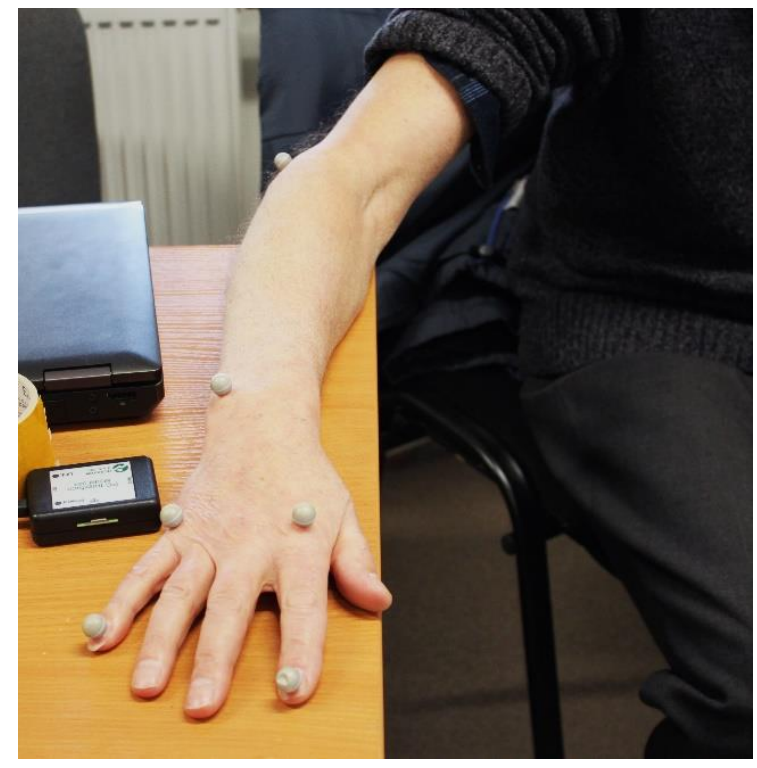

Fig. 2 Markers on the arm of tremor investigation points

Cameras captured markers' position and accelerations. Accelerations were processed to get tremor frequency graphs while positions - to get trajectories of tremor.

To analyze difference between resting tremor and postural tremor parameters, subjects were asked to hold various stances in front of cameras: outstretched arm with palm horizontally to ground, outstretched arm with palm vertically to ground, totally relaxed arm, relaxed arm on the knee while sitting, vertically held arm. Tremors in different stances were analyzed in 1 minute periods with breaks between the measurements.

Raw values of hand position were processed with MatLab 2014b software. To obtain tremor trajectories, data were filtered with $2 \mathrm{~Hz}$ high pass and $10 \mathrm{~Hz}$ low pass Finite Impulse Response (FIR) filters (1):

$$
y(n)=\sum_{m=0}^{M} h(m) x(n-m),
$$

where $h$ is impulse response coefficient, $M$ is filter order, $x(n-m)$ is input signal delayed by $m$. This filter doesn't use feedback therefore requires more computational resources, but FIR filter is always stable, as all poles are located at the origin. Signals of natural hand movements were removed by using high pass filter while the low pass filtered worked as anti-aliasing filter and removed noise from the signal. 2 -
$10 \mathrm{~Hz}$ range was chosen based on the frequency analysis and studies that disclosed the range of hand tremor frequencies.

Device that uses mechanical vibrations to increase blood circulation (ViLim) was used to test the interference with hand tremor [10]. Data of tremor oscillations was compared, analyzed and concluded with device turned on and off. Measurements with gyroscope were conducted to see its effect for hand tremor.

ViLim uses electric motors that creates mechanical vibrations of different frequencies. This low-high frequency combination is needed to use beating phenomenon [11] that creates oscillations of low frequency. To ensure low frequency vibrations and higher than $1 \mathrm{~mm}$ limb amplitude, the beating phenomenon has been considered as the most appropriate. Otherwise, high voltage and heavy motors have to be selected in the case of aspiration of low-frequencies. By using complex algorithms and implementing real time tracking systems hand tremor stabilization can be achieved. Our goal is to use ViLim to counter hand tremor movement. Several researches have been implemented in purpose to develop tremor sensing or mitigation systems verifying possibility of achieving this goal. Researchers from Malaysia's University of Technology have employed piezoelectric actuator with active force control technique to control human hand tremor in simulation [12]. Robert LeMoyne et al. used wireless accelerometers to collect tremor parameters data from 20 trials [13]. Same parameters can be gathered by using gyroscopes, haptic devices, etc. [14]. Accelerometers could be used to separate voluntary and involuntary motions from raw data as well [15].

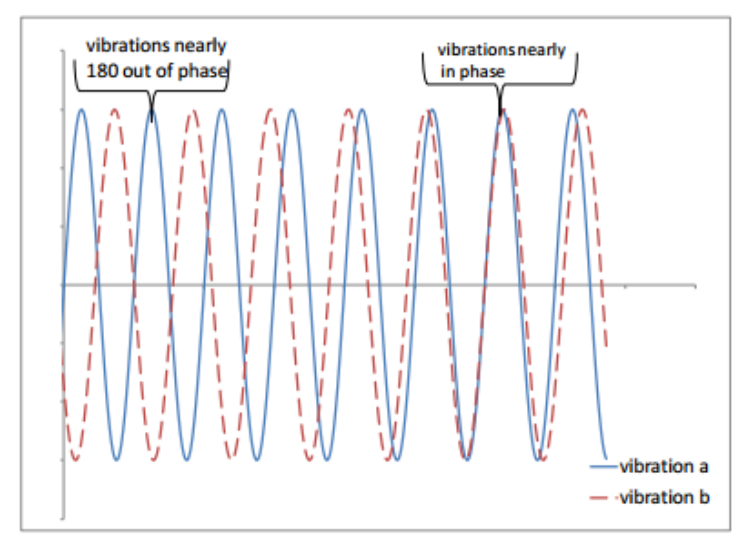

Fig. 3 Beating phenomenon occurrence [11]

\section{Results}

We analysed travelled distance of markers during the period of $54 \mathrm{~s}$ (100 Hz sampling frequency) of recording (Fig. 4). Different patients showed different travelled distances, but the pattern remained the same. Longest distances were travelled in furthest points of the limb - points on the nails of index and small finger. It was followed by points of knuckles of small and index finger. Movement range of wrist, elbow and shoulder were the smallest comparing with other points. After processing and analysing the data, decision was made to put device after the wrist, where the tremor has the biggest effect. 




Fig. 4 Markers travelled distance during $54 \mathrm{~s}$ recording

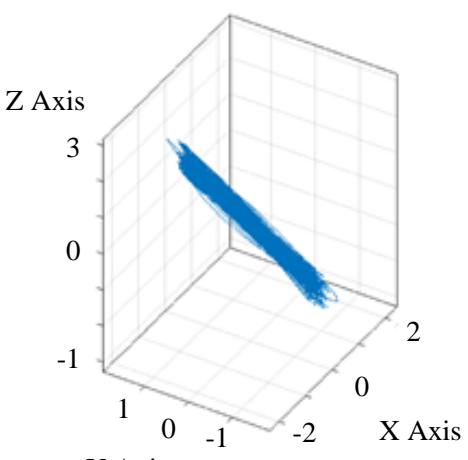

Y Axis
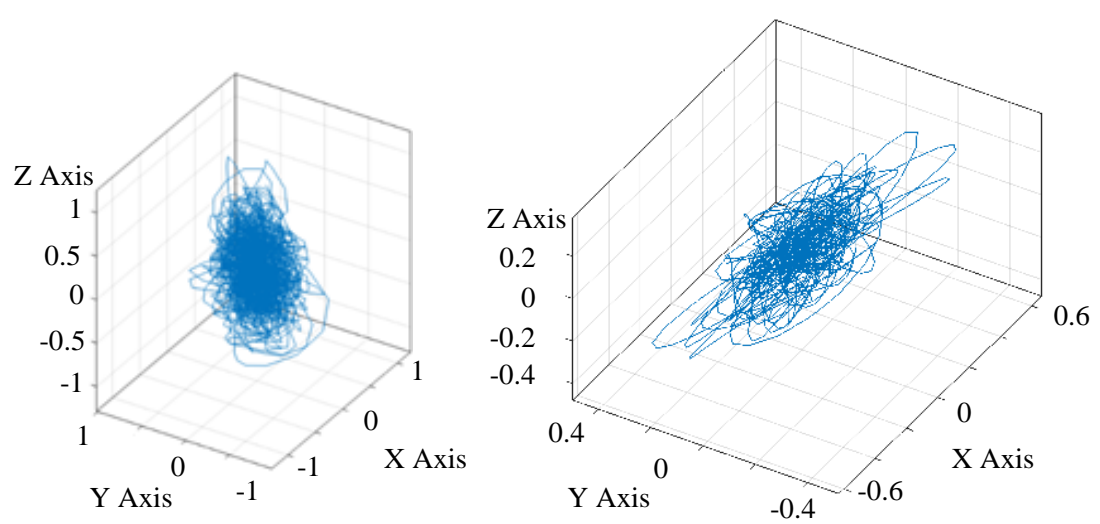

Fig. 5 Hand tremor data gathered from marker on finger and filtered with $10 \mathrm{~Hz}$ low pass and $2 \mathrm{~Hz}$ high pass FIR filters to get tremor trajectory. Left and centre: trajectory from patients. Right: trajectory created with device. Note: device trajectory can be rotated independently from axes

For each patient trajectory varies, nevertheless the rotational movement can be seen in every of them and the tremor shape can be described as ellipsoidal. Healthy person's hand with device turned on creates similar trajectory. These results are shown in Fig. 5.



Fig. 6 Tremor frequency on $\mathrm{x}, \mathrm{y}, \mathrm{z}$ axis

Fast Fourier transform [16] were used to find the main frequencies of the gathered signal that is shown in Fig. 6 and Fig. 7. Main frequency range was observed in the range of $4-8 \mathrm{~Hz}$. In one case (Fig. 7), there were second noticeable frequency of $9.5 \mathrm{~Hz}$, with smaller amplitude. Fig. 8 shows spectrogram of index finger nail in all axes and there the main frequency with some small amplitude harmonics could be clearly seen. Frequency varies from $0.1 \mathrm{~Hz}$ to $0.5 \mathrm{~Hz}$ when changing stances. There was no connection found between frequency changes when changing stances.

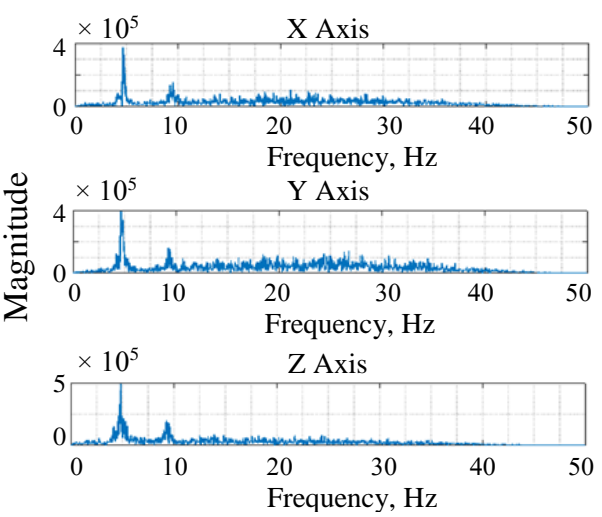

Fig. 7 Main and visible second tremor frequencies on $x, y, z$ axis

Fig. 9 shows the amplitudes of the filtered hand tremor signal when hand is held straight with palm horizontally. Typical amplitude is $2 \mathrm{~mm}$, but it varies from $1 \mathrm{~mm}$ to $4 \mathrm{~mm}$. Sampling frequency is $100 \mathrm{~Hz}$. $6 \mathrm{~Hz}$ were seen on the patient hand and $6 \mathrm{~Hz}$ were created by external mechanical oscillations source. Filtered tremor amplitude captured 

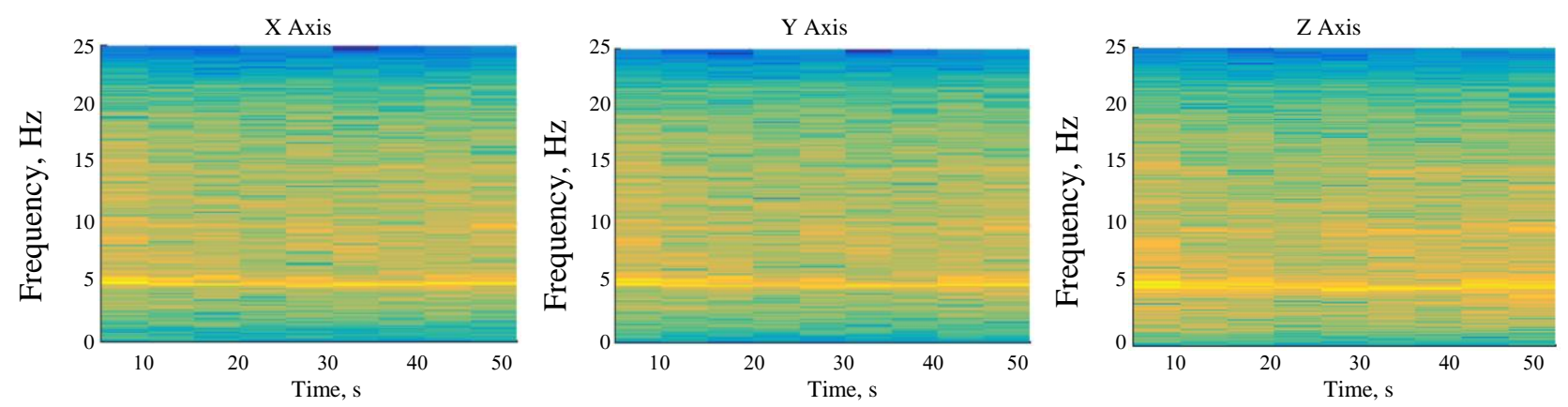

Fig. 8 Hand tremor spectrogram with $5 \mathrm{~Hz}$ main frequency on $\mathrm{X}, \mathrm{Y}, \mathrm{Z}$ axis from left to right
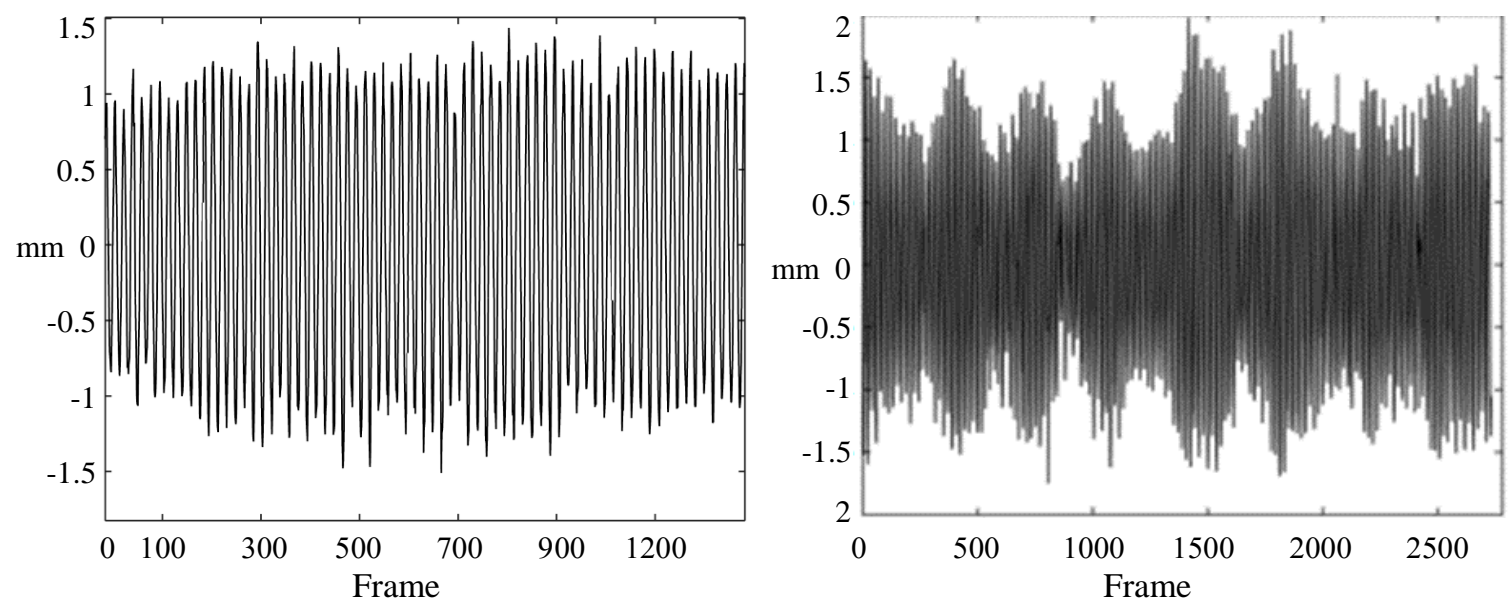

Fig. 9 Left: filtered hand tremor when hand is held horizontally straight and device is off. Right: filtered hand tremor when hand is held horizontally straight and device is on

from small finger nail with device turned off is shown on the left graph while the right graph shows filtered tremor amplitude captured from small finger nail with device turned on. Device created vibrations interferes with hand tremor vibrations and both vibrations are summed up. In the graph can be seen that minimum and maximum values (oscillations) appear when the device is on, due to misaligned phase of the vibrations and slight variation of hand tremor frequency. Maximum values appear $50 \%$ greater than stable tremor amplitude and minimum values were $16.5 \%$ lower than stable tremor amplitude. These results show the compatibility of the method but the control algorithm needs to be improved. In the further steps, sensors for momentum diagnostic of hand's frequency are planned to be installed into the same device. This will enable to reduce response time and simplify interference of hand and device vibrations.

Gyroscope effect to tremor was also tested resulting in elimination of rotary tremor motion. Natural hand movements were also affected. Subjects felt uncomfortable to move arms as gyroscope reduce all rotary movements including voluntary ones

\section{Conclusions}

1. An experimental analysis of hand tremor vibrations were made and tremor interference with external mechanical oscillations source was tested.

2. The experiments showed that the main frequencies of the hand tremor varies from 4 to $8 \mathrm{~Hz}$, most commonly $\sim 5 \mathrm{~Hz}$. In one case second frequency appeared $(9,5$ $\mathrm{Hz}$ ). Amplitude varies from 2 to $5 \mathrm{~mm}$ on index and small finger nails and amplitude decreases when moving to shoulder. Tremor trajectory shape is always ellipsoidal.
3. Interference with external mechanical oscillations source ViLim reduced vibration amplitude $16.5 \%$ on interference minimum and increased up to $48.8 \%$ on interference maximum concluding that tremor can be affected by external mechanical oscillations.

4. By mounting gyroscope on the hand, we noticed reduction of hand tremor amplitude as well as impairment of natural hand movements.

5. Further analysis are focused on improvement of the algorithm which will identify and process hand movements and creates mechanical response based of tremor frequency and amplitude. This improvement will increase device efficiency and hand tremors will be reduced to some point, but not completely

\section{Acknowledgement}

This research was funded by a grant (No. SEN10/15), from the Research Council of Lithuania.

\section{References}

1. Müller, Thomas. 2012. Drug therapy in patients with Parkinson's disease, Translational Neurodegeneration 1(1): 1 . http://dx.doi.org/10.1186/2047-9158-1-10.

2. Pathak, Anupam, et al. 2014. A noninvasive handheld assistive device to accommodate essential tremor: a pilot study, Movement Disorders 29(6): 838-842. http://dx.doi.org/10.1002/mds.25796.

3. Hand-held gyroscopic device US $5058571 \mathrm{~A}$.

4. Adjustable and tunable hand tremor stabilizer US 6730049 B2. 
5. Rocon, Eduardo, et al. 2007. Mechanical suppression of essential tremor, The Cerebellum 6(1): 73-78. http://dx.doi.org/10.1080/14734220601103037.

6. Yang, Jen-Lin, et al. 2016. Detection of hand tremor in patients with Parkinson's disease using a non-invasive laser line triangulation measurement method, Measurement 2(79): 20-28. http://dx.doi.org/10.1016/j.measurement.2015.10.028.

7. Pledgie, Stephen, et al. 2000. Tremor suppression through impedance control, IEEE Transactions on Rehabilitation Engineering 8(1): 53-59. http://dx.doi.org/10.1109/86.830949.

8. Meshack, R. P.; Norman, K. E. 2002. A randomized controlled trial of the effects of weights on amplitude and frequency of postural hand tremor in people with Parkinson's disease, Clinical Rehabilitation 16(5): 481492. http://dx.doi.org/10.1191/0269215502cr521oa.

9. Benevicius, Vincas; Ostasevicius, Vytautas; Gaidys, Rimvydas. 2013. Identification of capacitive MEMS accelerometer structure parameters for human body dynamics measurements, Sensors 13(9): 11184-11195. http://dx.doi.org/10.3390/s130911184.

10. Venslauskas, Mantas. 2015. Investigation and application of the human blood flow improvement by mechanical vibrations. PhD dissertation, Institute of Mechatronics, Kaunas University of Technology.

11. Yalla, S.K.; Kareem, A. 2000. On the beat phenomenon in coupled systems, Proceedings of the 8th ASCE Joint Specialty Conference on Probabilistic Mechanics and Structural Reliability, Notre Dame, Indiana, July 24-26, 2000.

12. Kazi, Suhail; Mailah, Musa; Zain, Zarhamdy MD. 2014. Suppression of hand postural tremor via active force control method, Proceedings of the 14th International Conference on Robotics, Control and Manufacturing Technology.

13. LeMoyne, Robert; Mastroianni, Timothyand; Grundfest, Warren. 2013. Wireless accelerometer configuration for monitoring Parkinson's disease hand tremor, Advances in Parkinson's Disease 2(2): 62-67. http://dx.doi.org/10.4236/apd.2013.22012.

14. Grimaldi, Giuliana; Manto, Mario. 2010. Neurological tremor: Sensors, signal processing and emerging applications, Sensors 10(2): 1399-1422. http://dx.doi.org/10.3390/s100201399.

15. Veluvolu, Kalyana C.; Ang, Wei Tech. 2011. Estimation of physiological tremor from accelerometers for real-time applications, Sensors 11(3): 3020-3036. http://dx.doi.org/10.3390/s110303020.

16. Orfanidis, Sophocles J. 1995. Introduction to Signal Processing. Prentice-Hall, Inc.
M. Venslauskas, E. Litvinas, A. R. Juknevičius, V. Ostaševičius

\section{RESEARCH OF HAND TREMOR VIBRATIONS AND INTERFERENCE WITH EXTERNAL MECHANICAL OSCILLATIONS SOURCE}

S u m m a r y

This paper presents the research of hand tremor vibrations and interference with external mechanical vibrations source. Photogrammetry were used to capture and analyze hand tremor parameters from 8 patient with essential and Parkinson tremors. Blood perfusion device, that creates mechanical vibrations, was used as an external source of mechanical vibrations and was tested with patients. Device interference with hand tremor showed promising results of hand tremor reduction. The minimum points had $16.5 \%$ lower amplitude of hand tremor. The maximum amplitudes were about $50 \%$ greater and these results show the compatibility of the method but the control algorithm needs to be improved.

Keywords: mechanical vibrations, tremor, Parkinson's disease.

Received June 01, 2016

Accepted September 28, 2016 